\title{
Nearest Optimal Repeatable Control Strategies for Kinematically Redundant Manipulators
}

\author{
Rodney G. Roberts, Student Member, IEEE, and Anthony A. Maciejewski, Member, IEEE
}

\begin{abstract}
Kinematically redundant manipulators, by definition, possess an infinite number of generalized inverse control strategies for solving the Jacobian equation. These control strategies are not, in general, repeatable in the sense that closed trajectories for the end-effector do not result in closed trajectories in the joint space. The Lie bracket condition (LBC) can be used to check for the possibility of integral surfaces, also called stable surfaces, which define regions of repeatable behavior. However, the LBC is only a necessary condition. In this work, a necessary and sufficient condition for the existence of stable surfaces is used to illustrate that such surfaces are much rarer than previously thought. This motivates the main result of this work, which is a technique for designing a repeatable control that is nearest, in an integral norm sense, to a desired optimal control. The desired optimal control is allowed to take the form of any generalized inverse. An example is presented that illustrates the capability of designing repeatable controls that approximate the behavior of desired optimal inverses in selected regions of the workspace.
\end{abstract}

\section{INTRODUCTION}

$\mathbf{K}^{\prime}$ INEMATICALLY redundant manipulators are robotic systems that possess more degrees of freedom than are required to perform a specified task. For single-arm manipulators the task is usually specified as a location or path for the end-effector. A manipulator can be described by its kinematic equation

$$
x=f(\theta)
$$

where $x \in \mathbb{R}^{m}$ represents the workspace position and/or orientation of the end-effector and $\theta \in \mathbb{R}^{n}$ represents the manipulator's joint configuration. Thus, $m<n$ by definition for redundant manipulators. The Jacobian equation relates the joint velocities to the end-effector velocities and, for the positional component, is obtained by differentiating (1) resulting in

$$
\dot{\boldsymbol{x}}=J \dot{\theta} .
$$

Due to the extra degrees of freedom, redundant manipulators possess an infinite number of local control schemes of the form

$$
\dot{\theta}=G \dot{x}
$$

where $J G=I$ (except at singularities of $J$ or possibly $G$ ) in order to satisfy the constraint of a given end-effector velocity.

Manuscript received April 10, 1991; revised October 17, 1991. This work was supported by Sandia National Laboratories under Contract 18-4379B. Additional support was provided by the NEC Corporation and the TRW Foundation. Portions of this paper were presented at the IEEE Systems, Man. and Cybernetics Conference, Charlottesville, VA, October 13-16, 1991.

The authors are with the School of Electrical Engineering, Purdue University, West Lafayette, IN 47907.

IEEE Log Number 9107142.
A popular local control scheme is pseudoinverse control due to its desirable minimum norm property.

A generalized inverse control like the one given in (3) may not be repeatable in the sense that closed trajectories in the workspace may not be mapped to closed trajectories in the joint space. Pseudoinverse control is no exception as Klein and Huang [3] have shown. When a cyclic task is performed using a nonrepeatable control, the joint angles of the manipulator do not necessarily return to their initial position. In other words, generalized inverse control of kinematically redundant manipulators may produce a drift in joint space when a cyclic task is performed in the workspace. This may pose a problem since the manipulator's behavior would be hard to predict without prior analysis. By using a repeatable control, the setup time for a manipulator can be reduced for cyclic tasks since one would only need to check one cycle to see if the manipulator functioned as desired.

Shamir and Yomdin [9] have developed an elegant test using Frobenius's theorem from differential geometry for determining whether or not an arbitrary inverse is repeatable on an open subset of the joint space. This test, called the Lie bracket condition (LBC), is formulated in terms of the Lie bracket of the columns of the inverse. The Lie bracket of two vectors $\boldsymbol{u}$ and $\boldsymbol{v}$, where both vectors are functions of $\theta$, is given by the vector

$$
[\boldsymbol{u}, \boldsymbol{v}]=\left(\frac{\partial \boldsymbol{v}}{\partial \theta}\right) \boldsymbol{u}-\left(\frac{\partial \boldsymbol{u}}{\partial \theta}\right) \boldsymbol{v}
$$

An inverse $G$ is said to satisfy the LBC if the Lie bracket of any two columns of $G$ is in the column space of $G$. A necessary and sufficient condition for a control strategy to be repeatable on an open singularity-free region of the joint space is that the LBC hold on this region. For the special case of the pseudoinverse one need only apply the LBC to $J^{T}$, which greatly simplifies the computations required (see Appendix I). The LBC also gives necessary conditions for what are called stable surfaces, which are defined as $m$-dimensional hypersurfaces in the joint space on which the control strategy exhibits repeatable behavior. When a manipulator starts initially on a stable surface, the manipulator will continue to remain on this surface except possibly when encountering a singular configuration. An example is given in [9] where the stable surfaces for the pseudoinverse solution of a three-link planar manipulator described in absolute joint angles are calculated. It is important to note that the LBC is a necessary but not sufficient condition for testing whether or not a surface is a stable surface. A surface that satisfies the 
LBC but that is not necessarily a stable surface will be called a candidate surface. An example illustrating the significance of this distinction is presented in the following section.

The remainder of this paper is organized in the following manner: Section II discusses a necessary and sufficient condition for a candidate surface to be a stable surface for planar three-link manipulators [8]. Some of the difficulties in identifying such surfaces are illustrated. In particular, it is shown that a commonly analyzed manipulator that had previously been thought to possess stable surfaces does not in fact have any such surfaces. In Section III, the LBC is used to define a class of repeatable solutions. After a class of repeatable solutions is found, a technique for determining the member of this class nearest to a desired inverse in an integral norm sense is presented. Section IV then illustrates this design technique with a specific example. Simulation results are then presented in Section V with conclusions appearing in Section VI.

\section{Stable Surfaces}

As pointed out in [8] and [9], even if the $\mathrm{LBC}$ is satisfied for a given surface, the surface is not necessarily a stable surface. For planar three-link manipulators, there is a simple sufficiency condition for stable surface candidates. If using a control strategy that has the form of (3), the LBC specifies that a candidate surface must satisfy the equation

$$
\boldsymbol{n}_{G}^{T}\left[\boldsymbol{g}_{1}, \boldsymbol{g}_{2}\right]=0
$$

where $G=\left[\boldsymbol{g}_{1} \boldsymbol{g}_{2}\right]$ and $\boldsymbol{n}_{G}$ is a null vector of $G^{T}$. It can be easily shown (see Appendix II) that (5) is equivalent to

$$
\boldsymbol{n}_{G} \cdot \nabla \times \boldsymbol{n}_{G}=0
$$

which is the curl condition used in [3]. Thus, for planar threelink manipulators, candidate surfaces must satisfy $s(\theta)=\boldsymbol{n}_{G}$. $\nabla \times \boldsymbol{n}_{G}=0$. For the special case where $G$ is the pseudoinverse, a candidate surface must satisfy $s(\theta)=\boldsymbol{n}_{J} \cdot \nabla \times \boldsymbol{n}_{J}=0$ where $\boldsymbol{n}_{J}$ is a vector in the null space of $J$.

Now suppose that this manipulator has a stable surface $S$ and that this surface is described by the equation $s(\theta)=0$. If the manipulator is on this surface $S$, then for any specified end-effector trajectory the following must hold:

$$
\dot{s}=\sum_{i=1}^{n} \frac{\partial s}{\partial \theta_{i}} \dot{\theta}_{i}=\nabla s \cdot \dot{\theta}=0 .
$$

Using the fact that $\dot{\theta}=G \dot{\boldsymbol{x}}$, one obtains

$$
\nabla^{T} G \dot{\boldsymbol{x}}=0 .
$$

Since this is true for all $\dot{x}$

$$
\nabla^{T} s G=\mathbf{0}^{\mathrm{T}} .
$$

If the manipulator is not in a singular configuration, then (9) implies that $\nabla s\left(\theta_{s}\right)$ and $\boldsymbol{n}_{G}\left(\theta_{s}\right)$ are proportional for all $\theta_{s}$ on $S$. Thus, (9) provides a necessary and sufficient condition for identifying a stable surface for planar three-link manipulators [8].

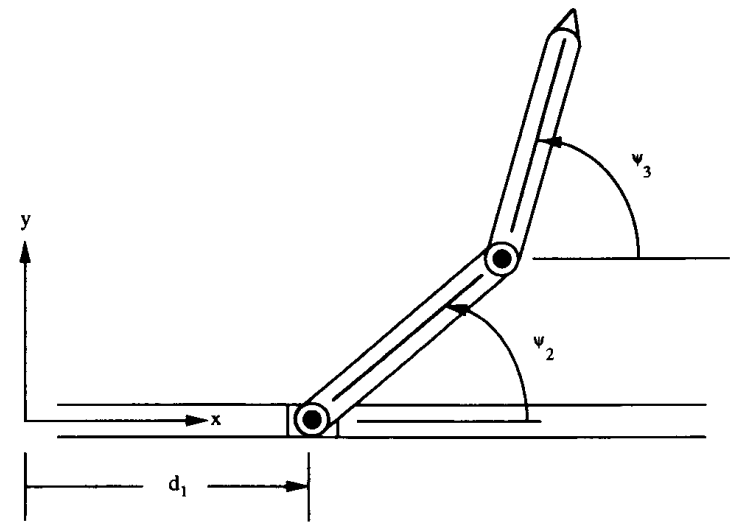

Fig. 1. Geometry of a planar three-link manipulator whose first joint is prismatic and whose last two joints are revolute and of unit link length. The rotary joints are described in terms of absolute angles.

As an illustration of this necessary and sufficient condition, consider the planar manipulator depicted in Fig. 1 with one prismatic joint and two revolute joints of unit length. This manipulator is described by the kinematic function

$$
\boldsymbol{x}=\boldsymbol{f}(\psi)=\left[\begin{array}{c}
d_{1}+\cos \psi_{2}+\cos \psi_{3} \\
\sin \psi_{2}+\sin \psi_{3}
\end{array}\right]
$$

where $\psi=\left[\begin{array}{lll}d_{1} & \psi_{2} & \psi_{3}\end{array}\right]^{T}$ and $\psi_{i}$ represents the absolute joint angle as opposed to the more commonly used relative joint angle. The manipulator Jacobian is then given by

$$
J=\left[\begin{array}{ccc}
1 & -\sin \psi_{2} & -\sin \psi_{3} \\
0 & \cos \psi_{2} & \cos \psi_{3}
\end{array}\right] .
$$

A null vector of the Jacobian can easily be calculated by taking the cross product of the two rows and is given by

$$
\boldsymbol{n}_{J}=\left[\begin{array}{c}
\sin \left(\psi_{3}-\psi_{2}\right) \\
-\cos \psi_{3} \\
\cos \psi_{2}
\end{array}\right] \text {. }
$$

A simple calculation shows that the necessary condition for a stable surface described by (6) results in

$$
s(\theta)=\boldsymbol{n}_{J} \cdot \nabla \times \boldsymbol{n}_{J}=\cos \psi_{3}-\cos \psi_{2}=0 .
$$

This condition is satisfied on the surfaces

$$
\psi_{2}=\psi_{3}
$$

and

$$
\psi_{2}=-\psi_{3}
$$

Applying the condition described by (9), the gradient of $s(\theta)$ on the first surface is proportional to the null vector $\boldsymbol{n}_{J}$ while on the second surface it is actually perpendicular to $\boldsymbol{n}_{J}$. Thus, (14) describes a stable surface, but the surface described by (15), while satisfying the LBC, is not a stable surface.

In fact, the existence of stable surfaces is not as common as previously thought. In the literature [9], stable surfaces have been used to explain the convergence of a cyclic task to a repeatable solution. It was claimed that, as a manipulator 
approaches a stable surface, the drift in the joint angles approaches zero. However, it will be shown through an example that convergence to a repeatable solution does not imply that the manipulator has a stable surface. This will be done by applying the LBC and (9) to the planar three-link revolute manipulator shown in Fig. 2. Note that the joint angles for this manipulator are expressed in relative angles as opposed to absolute angles. The Jacobian for this manipulator is given by

$J=$
$\left[\begin{array}{ccc}-\sin \theta_{1}-\sin \theta_{12}-\sin \theta_{123} & -\sin \theta_{12}-\sin \theta_{123} & -\sin \theta_{123} \\ \cos \theta_{1}+\cos \theta_{12}+\cos \theta_{123} & \cos \theta_{12}+\cos \theta_{123} & \cos \theta_{123}\end{array}\right]$

where $\theta_{i j}$ denotes $\theta_{i}+\theta_{j}$. In order for this manipulator to be on a stable surface, the following equation must be satisfied:

$$
s(\theta)=-\sin \theta_{2}+\sin \theta_{3} \cos \theta_{2}+\sin _{3} \cos \left(\theta_{2}+\theta_{3}\right)=0
$$

where $\theta_{i}$ are the relative joint angles. For this manipulator, a null vector is given by

$$
\boldsymbol{n}_{J}=\left[\begin{array}{c}
\sin \theta_{3} \\
-\sin \theta_{3}-\sin \left(\theta_{2}+\theta_{3}\right) \\
\sin \theta_{2}+\sin \left(\theta_{2}+\theta_{3}\right)
\end{array}\right]
$$

while the gradient of $s$ is

$$
\begin{aligned}
& \nabla s= \\
& {\left[\begin{array}{c}
0 \\
-\cos \theta_{2}-\sin \theta_{2} \sin \theta_{3}-\sin \theta_{3} \sin \left(\theta_{2}+\theta_{3}\right) \\
\cos \theta_{2} \cos \theta_{3}+\cos \theta_{3} \cos \left(\theta_{2}+\theta_{3}\right)-\sin \theta_{3} \sin \left(\theta_{2}+\theta_{3}\right)
\end{array}\right] .}
\end{aligned}
$$

Now in order for this manipulator to possess a stable surface, (9) must be satisfied, which can only occur when either $\boldsymbol{n}_{J}$ and $\nabla s$ are proportional or $\nabla s=0$. By comparing the first element of (18) with (19), it is easily seen that the first case can only occur when $\sin \theta_{3}=0$. If this is true, then from (17) it can be seen that $\sin \theta_{2}$ is also zero so that the manipulator is in a singular configuration. Now consider the case where $\nabla s=\mathbf{0}$. One can show that the second element of $\nabla s$ cannot be zero on a surface described by $s$ by noting that

$$
s^{2}+\left(\frac{\partial s}{\partial \theta_{2}}\right)^{2}=1+2 \sin ^{2} \theta_{3}\left(2+\cos \theta_{3}\right)>0
$$

where $\partial s / \partial \theta_{2}$ is the second element of $\nabla s$. Since $s^{2}+$ $\left(\partial s / \partial \theta_{2}\right)^{2}>0$, there is no value of $\theta$ for which $s=0$ and $\nabla s=0$. Thus, the two surfaces satisfying $s=0$ are not stable surfaces. Therefore, the convergence behavior noted in [3] and [4] is convergence to a repeatable solution for a given closed trajectory as opposed to convergence to a stable surface. Since this manipulator is known to possess a stable surface when described in terms of absolute angles [9], this example illustrates the important point that stable surfaces are not preserved under joint transformations. Thus, one cannot simply warp an existing surface by changing the weighting matrix on the pseudoinverse.

A geometrical interpretation of the constraints imposed by the LBC illustrates why one should intuitively consider

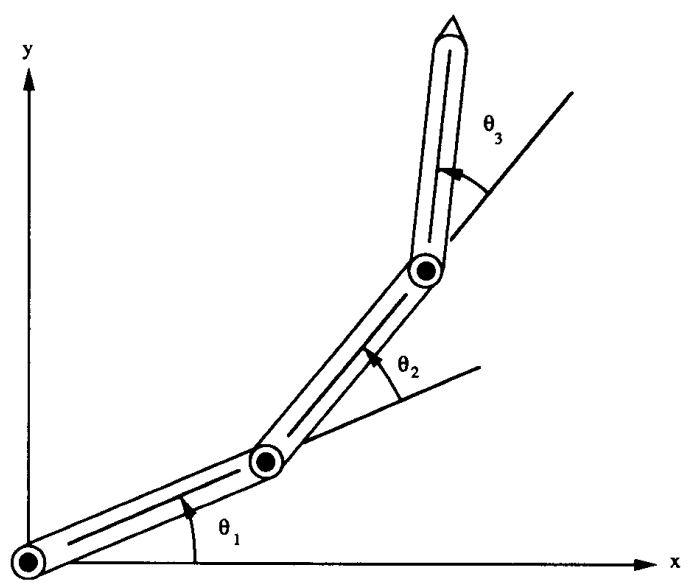

Fig. 2. Geometry of a planar three-link manipulator whose joints are all revolute and of unit link length. The joint angles are described in terms of relative angles.

stable surfaces to be the exception rather than the rule. For a stable surface to exist, all of the hypersurfaces defined by the constraint equations obtained from the $\mathrm{LBC}$ must all intersect on the same hypersurface of appropriate dimension. While one can construct an infinite number of such examples, they are typically highly restricted cases of a general manipulator. For example, consider the generalization of the planar $3 \mathrm{R}$ manipulator in [9] to a general $n$-link planar manipulator described in absolute angles. By applying (9) one can show that stable surfaces will only exist under the restriction that there are at most two distinct link lengths. On such surfaces there will only be two distinct joint values. Physically this means that all but two of the joints are frozen so that, in effect, all such manipulators behave as planar two-link manipulators. Note that for all practical purposes one could never even manufacture such a manipulator since the link lengths could never be guaranteed to be exactly equal.

\section{A Class of RePEATABle INVERSES}

The previous section has shown that one cannot rely on the existence of stable surfaces to achieve repeatability. An alternative, which is illustrated in this section, is to derive a class of repeatable solutions. These inverses, like the extended Jacobian [1], have foliations of stable surfaces and so are guaranteed to be repeatable. The inverses under consideration are generalized inverses like those described by (3). For a manipulator with a single degree of redundancy, any of these inverses can be written in the form

$$
G=J^{+}+\hat{n}_{J} \boldsymbol{w}^{T}
$$

where $\hat{\boldsymbol{n}}_{J}$ is the unit length null vector of the Jacobian $J$ and $\boldsymbol{w}$ is a vector that uniquely determines $G$. This follows from the fact that $J\left(G-J^{+}\right)=[0]$. Similarly, for higher degrees of redundancy

$$
G=J^{+}+\left[\begin{array}{lll}
\hat{\boldsymbol{n}}_{1} & \cdots & \hat{\boldsymbol{n}}_{n-m}
\end{array}\right]\left[\begin{array}{c}
\boldsymbol{w}_{1}^{T} \\
\vdots \\
\boldsymbol{w}_{n-m}^{T}
\end{array}\right]
$$


where $\hat{\boldsymbol{n}}_{1}, \ldots, \hat{\boldsymbol{n}}_{n-m}$ form an orthonormal basis for the null space of $J$. For simplicity, only the case of a single degree of redundancy will be considered unless otherwise noted. From (21), it is easy to verify that

$$
\boldsymbol{n}_{G}=J^{T} \boldsymbol{w}-\hat{\boldsymbol{n}}_{J}
$$

is a null vector of $G^{T}$. The first step in designing an optimal repeatable inverse is to determine the vector functions $w$ that characterize the set of repeatable inverses. This can be done by determining all of the $\boldsymbol{w}$ that satisfy the differential equations given by

$$
\boldsymbol{n}_{G}^{T}\left[\boldsymbol{g}_{i}, \boldsymbol{g}_{j}\right]=0, \quad 1 \leq i<j \leq m .
$$

For three-link planar manipulators, this set of equations can be simplified by using the equivalent form

$$
\boldsymbol{n}_{G} \cdot \nabla \times \boldsymbol{n}_{G}=0 .
$$

Equation (24), or (25) where applicable, determines a class of admissible $\boldsymbol{w}$ so that for any $\boldsymbol{w}$ in this class, the corresponding inverse $G_{r}$ is repeatable.

There are infinitely many such repeatable inverses so that it is possible to optimize over this class in order to obtain additional desirable properties. One possible approach is to minimize the distance to an unrepeatable inverse $G_{d}$ that possesses some desirable characteristics. The measure that will be used in this work is

$$
\int_{\Omega}\left\|G_{r}-G_{d}\right\|_{2}^{2} d \theta
$$

where $\|\cdot\|_{2}$ is the Euclidean matrix norm. Equation (26) gives a measure of the closeness of the two inverses over the connected subset $\Omega$ of the joint space. Alternatively, one could integrate over a region of the workspace in order to determine the optimal foliation in the joint space. From (21) it follows that

$$
\begin{aligned}
\left\|G_{r}-G_{d}\right\|_{2} & =\left\|\left(J^{+}+\hat{\boldsymbol{n}}_{J} \boldsymbol{w}_{r}^{T}\right)-\left(J^{+}+\hat{\boldsymbol{n}}_{J} \boldsymbol{w}_{d}^{T}\right)\right\|_{2} \\
& =\left\|\hat{\boldsymbol{n}}_{J}\left(\boldsymbol{w}_{r}-\boldsymbol{w}_{d}\right)^{T}\right\|_{2}
\end{aligned}
$$

where $\boldsymbol{w}_{d}$ is the unique vector that corresponds to $G_{d}$. Since the Euclidean matrix norm of an outer product is equal to the product of the corresponding vector norms, it follows that

$$
\left\|G_{r}-G_{d}\right\|_{2}=\left\|\hat{\boldsymbol{n}}_{J}\right\|_{2}\left\|\boldsymbol{w}_{r}-\boldsymbol{w}_{d}\right\|_{2} .
$$

Since $\hat{\boldsymbol{n}}_{J}$ is of unit length, the optimization criterion becomes

$$
\int_{\Omega}\left\|G_{r}-G_{d}\right\|_{2}^{2} d \theta=\int_{\Omega}\left\|\boldsymbol{w}_{r}-\boldsymbol{w}_{\boldsymbol{d}}\right\|_{2}^{2} d \theta
$$

which greatly simplifies the computations.

In practice, solving for closed-form analytical solutions to a set of partial differential equations such as (24) may be a virtually impossible task. In order to find the nearest optimal repeatable control for more complicated manipulators, it is necessary to develop a method that does not rely on solving complicated differential equations. By using the known geometrical properties of repeatable inverses, it is possible to generate a subset of analytic solutions to these differential equations by utilizing gradient functions. While this technique has the advantage of avoiding complicated equations, its disadvantage is that it only optimizes over a subset of repeatable inverses. In order to apply this technique, a different characterization of the repeatable control strategies, which does not explicitly require the solution of equations resulting from the $\mathrm{LBC}$, is necessary. This method relies on characterizing the vectors that are at every value $\theta$ orthogonal to the joint trajectories determined by the control strategy. These vectors are given by the null space of the transpose of the generalized inverse, which, for essentially all repeatable generalized inverses, are determined by gradient functions [8]. Thus, these repeatable strategies can be obtained by inverting the square matrix

$$
J_{\boldsymbol{v}}=\left[\begin{array}{c}
J \\
\cdots \\
\boldsymbol{v}^{T}
\end{array}\right]
$$

where $\boldsymbol{v}$ is a gradient function that characterizes the repeatable generalized inverse. In other words, inverses obtained in this manner are automatically guaranteed to satisfy the LBC given by (24) without explicitly solving the associated differential equations. As shown by Baker and Wampler, these inverses correspond to inverse kinematic functions [2]. Note that $J_{v}$ is superficially of the same form as an augmented or extended Jacobian. Clearly, if one can identify an appropriate number of additional kinematic constraints that correspond to the desired use of the redundancy, augmenting the Jacobian with these equations is the method of choice for resolving the redundancy and automatically guaranteeing a repeatable solution [7]. Likewise, if the desired additional objective is strictly a function of $\theta$, Baillieul [1] has shown how one can construct an optimal $v$. However, it is important to point out that the technique presented here is distinct from both the augmented and extended Jacobian techniques since there may be no physically meaningful function of $\theta$ that describes the desired optimization criterion. In particular, the proposed technique is able to handle more general optimization criteria such as the minimum joint velocity norm solution obtained using the pseudoinverse, which will be used as an example in the remainder of this work.

The repeatable inverses described by (30) are calculated at nonsingular configurations by taking the first $m$ columns of

$$
J_{\boldsymbol{v}}^{-1}=\left[\begin{array}{lll}
G_{d}-\hat{\boldsymbol{n}}_{J} \frac{\boldsymbol{v}^{T} G_{d}}{\hat{\boldsymbol{n}}_{J} \cdot \boldsymbol{v}} & \vdots & \frac{\hat{\boldsymbol{n}}_{J}}{\hat{\boldsymbol{n}}_{J} \cdot \boldsymbol{v}}
\end{array}\right]
$$

where once again $G_{d}$ is some desired (but typically not repeatable) generalized inverse. Thus any repeatable strategy has the form

$$
G_{r}=G_{d}-\hat{\boldsymbol{n}}_{J} \frac{\boldsymbol{v}^{T} G_{d}}{\hat{\boldsymbol{n}}_{J} \cdot \boldsymbol{v}}
$$

where $v$ is a gradient function. From (32) it follows that $\boldsymbol{w}_{r}-\boldsymbol{w}_{\boldsymbol{d}}$ is given by

$$
\boldsymbol{w}_{r}-\boldsymbol{w}_{d}=\frac{G_{d}^{T} \boldsymbol{v}}{\hat{n}_{J} \cdot \boldsymbol{v}} .
$$

The values of $\theta$ that result in $\hat{\boldsymbol{n}}_{J}(\theta) \cdot \boldsymbol{v}(\theta)=0$, but correspond to nonsingular configurations of the Jacobian, are called algorithmic singularities. These singularities, which were first 
noted by Baillieu [1] in the case of the extended Jacobian, cause (33) to take on infinite values. The cost function, corresponding to (26), on a simply connected singularity-free subset $\Omega$ of the joint space is thus given by

$$
\left\|G_{r}-G_{d}\right\|_{\Omega}^{2}=\int_{\Omega} \frac{\left\|G_{d}^{T} \boldsymbol{v}\right\|_{2}^{2}}{\left(\hat{\boldsymbol{n}}_{J} \cdot \boldsymbol{v}\right)^{2}} d \theta
$$

An example of a set of augmenting vectors that yield repeatable control strategies is the span of $N$ linearly independent gradient functions $\left\{\boldsymbol{v}_{1}, \boldsymbol{v}_{2}, \ldots, \boldsymbol{v}_{N}\right\}$. For this case, the augmenting vectors would have the form

$$
\boldsymbol{v}=\sum_{i=1}^{N} a_{i} \boldsymbol{v}_{i}
$$

where each $a_{i}$ is a real constant. The fact that $v$ is a gradient follows from the linearity of the differential operator. Several considerations should be made in choosing such a basis. One should be careful to select the gradient functions to be linearly independent from the row space of the Jacobian since failure to do so will result in a singular augmented Jacobian. Second, it should be noted that all multiples of an augmenting vector result in the same control. Thus, choosing an optimal augmenting vector becomes a constrained optimization problem in which each augmenting vector is normalized. Such a normalization can be done, for example, by requiring that $\sum_{i=1}^{N} a_{i}^{2}=1$. Combining (34) and (35) yields

$$
\left\|G_{r}-G_{d}\right\|_{\Omega}^{2}=\int_{\Omega} \frac{\sum_{i=1}^{N} \sum_{j=1}^{N} a_{i} a_{j} \boldsymbol{v}_{i}^{T} G_{d} G_{d}^{T} \boldsymbol{v}_{j}}{\left(\sum_{k=1}^{N} a_{k} \hat{\boldsymbol{n}}_{J} \cdot \boldsymbol{v}\right)^{2}} d \theta
$$

which is a criterion function of the coefficients $a_{i}$. Since the augmenting vectors have been normalized, (36) represents an $(N-1)$-dimensional optimization that will typically be performed numerically to obtain the desired coefficients of $v$. While this may require a significant amount of computation, it is important to note that it is all off-line calculation.

One can also generalize the above technique to higher degrees of redundancy. In this case, the augmented Jacobian has the form

$$
J_{V}=\left[\begin{array}{c}
J \\
\cdots \\
V^{T}
\end{array}\right]
$$

where $V$ is a matrix of $n-m$ gradient vectors. Equation (33) generalizes to

$$
W_{r}-W_{d}=-G_{d}^{T} V\left(\hat{N}^{T} V\right)^{-1}
$$

where $\hat{N}$ is a matrix whose columns form an orthonormal basis for the null space of $J$. The optimization still requires the numerical integration of (36) except that one must now calculate a matrix norm in place of the vector norm. Clearly, this may result in a considerable computational burden; however, once again it is all off-line calculation.

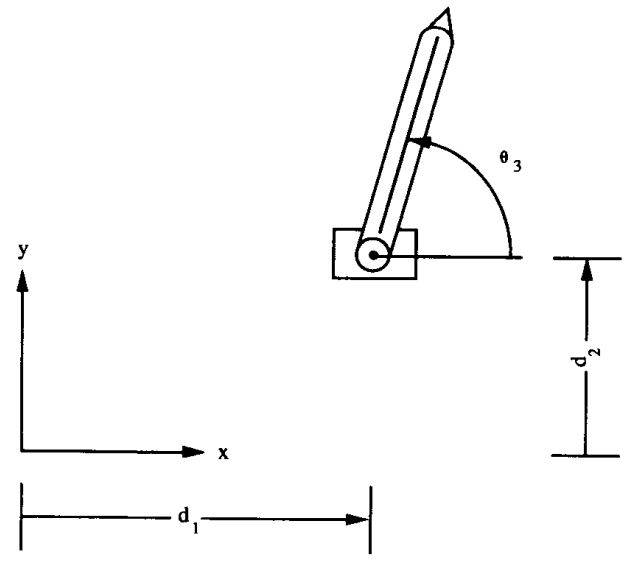

Fig. 3. Geometry of a planar three-link manipulator whose first two joints are prismatic and whose last joint is revolute and of unit link length.

\section{EXAMPLE}

In order to illustrate the ideas in the previous sections, a specific example will be presented. Consider the planar manipulator shown in Fig. 3 consisting of two orthogonal prismatic joints and a third revolute joint of unit length. The kinematic function for this manipulator is given by

$$
\boldsymbol{x}=\boldsymbol{f}(\theta)=\left[\begin{array}{l}
d_{1}+\cos \theta_{3} \\
d_{2}+\sin \theta_{3}
\end{array}\right]
$$

where $\boldsymbol{x}=\left[\begin{array}{ll}x & y\end{array}\right]^{T}$ and $\theta=\left[\begin{array}{lll}d_{1} & d_{2} & \theta_{3}\end{array}\right]^{T}$. It is easy to see that the Jacobian for this manipulator is

$$
J=\left[\begin{array}{ccc}
1 & 0 & -\sin \theta_{3} \\
0 & 1 & \cos \theta_{3}
\end{array}\right] .
$$

One can easily show that this simple manipulator possesses no stable surfaces when using pseudoinverse control [6]. In particular, the unit length null vector can be obtained by normalizing the cross product of the two rows of $J$ to yield

$$
\hat{\boldsymbol{n}}_{J}=\frac{1}{\sqrt{2}}\left[\begin{array}{c}
\sin \theta_{3} \\
-\cos \theta_{3} \\
1
\end{array}\right] \text {. }
$$

Testing the necessary condition for a stable surface, one obtains

$$
\hat{\boldsymbol{n}}_{J} \cdot \nabla \times \hat{\boldsymbol{n}}_{J}=-1 / 2
$$

thus proving that there are no stable surfaces. Therefore, no strategy that relies on convergence to a stable surface can be applied when a minimum norm solution is desired. However, the technique presented in Section III can be applied to achieve repeatable solutions and still retain some of the minimum norm properties of the pseudoinverse solution.

The first step in determining the repeatable inverse that is closest to the pseudoinverse is to characterize all generalized inverses by their null vectors. By using (23) these vectors can be written as

$$
\boldsymbol{n}_{G}=\left[\begin{array}{c}
w_{1}-\frac{1}{\sqrt{2}} \sin \theta_{3} \\
w_{2}+\frac{1}{\sqrt{2}} \cos \theta_{3} \\
-w_{1} \sin \theta_{3}+\mathrm{w}_{2} \cos \theta_{3}-\frac{1}{\sqrt{2}}
\end{array}\right]
$$


where they are now parameterized by $w_{1}$ and $w_{2}$. Next, these null vectors are restricted to those null vectors that correspond to a subset of repeatable inverses. This can be done by determining solutions to the differential equations obtained from (25) and (43). For this particular example, the solution of these equations is characterized by the relation

$$
w_{1}=k w_{2}+\frac{1}{\sqrt{2}}\left(\sin \theta_{3}+k \cos \theta_{3}\right)
$$

where $k$ is an arbitrary constant and it is assumed that the inverses are only function of $\theta_{3}$. Thus, (44) parameterizes these repeatable inverses in terms of the function $w_{2}$ and the constant $k$.

Now that a class of repeatable inverses has been derived, the optimal member of this class with respect to an appropriately chosen criterion function can be found. For this example the criterion function is given by

$$
C(k)=\int_{a}^{b}\left\|G_{r}-J^{+}\right\|_{2}^{2} d \theta_{3}
$$

which is a measure of the distance from the repeatable inverse $G_{r}$ to the pseudoinverse in the region $a \leq \theta_{3} \leq b$. It follows from (28) that

$$
\left\|G_{r}-J^{+}\right\|_{2}^{2}=\|\boldsymbol{w}\|_{2}^{2}=w_{1}^{2}+w_{2}^{2}
$$

so that minimizing $w_{1}^{2}+w_{2}^{2}$ is required. Using (44) and completing the square gives

$$
\begin{aligned}
w_{1}^{2}+w_{2}^{2}=\left(k^{2}+1\right)\left[w_{2}+\frac{k\left(\sin \theta_{3}+k \cos \theta_{3}\right)}{\sqrt{2}\left(k^{2}+1\right)}\right]^{2} \\
+\frac{\left(\sin \theta_{3}+k \cos \theta_{3}\right)^{2}}{\left(k^{2}+1\right)} .
\end{aligned}
$$

Now there is nothing that can be done about the last term of (47); however, the first term can be minimized by setting

$$
w_{2}=-\frac{k\left(\sin \theta_{3}+k \cos \theta_{3}\right)}{\sqrt{2}\left(k^{2}+1\right)}
$$

which results in

$$
w_{1}=\frac{k \cos \theta_{3}+\sin \theta_{3}}{\sqrt{2}\left(k^{2}+1\right)} .
$$

Equations (48) and (49) give a family of repeatable inverses, parameterized by $k$, that minimize (46). Substituting (48) and (49) into (46) gives

$$
\|\boldsymbol{w}\|_{2}^{2}=\frac{\left(\sin \theta_{3}+k \cos \theta_{3}\right)^{2}}{2\left(k^{2}+1\right)}
$$

which is bounded by $1 / 2$ thus insuring that the criterion function is well defined. Therefore, (45) becomes

$$
C(k)=\int_{a}^{b} \frac{\left(\sin \theta_{3}+k \cos \theta_{3}\right)^{2}}{2\left(k^{2}+1\right)} d \theta_{3} .
$$

Note that the optimization resulting in (48) and (49) is independent of $a$ and $b$, the limits of integration for (51).

The final form of the inverse that minimizes the cost function given by (51) depends on the limits of integration. This cost function when evaluated over an interval of length
TABLE I

Optimal Parameters for Symmetric Joint Limits $\left(C_{1}=0\right)$

\begin{tabular}{ccccc}
\hline & $0<b<\frac{\pi}{2}$ & $b=\frac{\pi}{2}$ & $\frac{\pi}{2}<b<\pi$ & $b=\pi$ \\
\hline$C_{2}$ & - & 0 & + & 0 \\
$k_{20 \mathrm{pt}}$ & 0 & any value & $\infty$ & any value \\
$\omega_{1}$ & $\frac{1}{\sqrt{2}} \sin \theta_{3}$ & equation (49) & 0 & equation (49) \\
$\omega_{2}$ & 0 & equation (48) & $-\frac{1}{\sqrt{2}} \cos \theta_{3}$ & equation (48)
\end{tabular}

$2 \pi$ has a value of $\pi / 4$ for all $k$ so that all choices of $k$ result in the same cost. However, one will typically want to restrict the limits of integration in order to obtain a closer match to the desired minimum norm solution in select configurations since the global constraint of repeatability significantly restricts the region in which the pseudoinverse can be approximated. To more clearly illustrate the effect of the limits of integration on the optimal solution, (51) is rewritten as

$$
C(k)=\frac{C_{1} k+C_{2}}{2\left(k^{2}+1\right)}+C_{3}
$$

where

$$
\begin{aligned}
& C_{1}=\frac{1}{2}(\cos 2 a-\cos 2 b) \\
& C_{2}=\frac{1}{2}(\sin 2 a-\sin 2 b)
\end{aligned}
$$

and

$$
C_{3}=\frac{1}{4}\left(b-a-C_{2}\right) .
$$

It can be easily shown that if $C_{1}$ is nonzero, the optimal choice for $k$ is

$$
k=\frac{C_{2}-\sqrt{C_{1}^{2}+C_{2}^{2}}}{C_{1}} .
$$

When $C_{1}=0$ the optimal choice for $k$ breaks down into two special cases, namely, $k=0$ and $k=\infty$. This will occur, for example, when the joint limits are symmetric, i.e., when $a=-b$, which is summarized in Table I. Note that for this case when $0<b<\pi / 2$ the inverse $G$ differs from the pseudoinverse only in the first column while for $\pi / 2<b<\pi$ the difference is only in the second column. This clearly illustrates the effect that the specified end-effector trajectory has on the difference between the repeatable solution and the minimum norm solution.

It is important to note that the optimal inverse need not be unique as is illustrated in Table I for the cases $b=\pi / 2$ and $b=\pi$ where any value of $k$ will result in an optimal solution. For this particular example, $k$ gives a parameterization of the optimal solutions in terms of (48) and (49) as $k$ varies over $\mathbb{R}$. In such cases where the optimal is not unique, it is possible to use some additional criterion to choose a specific solution. One possibility is to choose the optimal solution that is the least computationally expensive to implement. So for this particular example, the solution $k=0$ is selected to simplify the computation of (48) and (49) 
It is also possible to do the optimization in a different way. The criterion function $C(k)$ can be rewritten as

$$
C(k)=\int_{a}^{b} \frac{\left(\sin \theta_{3}+k \cos \theta_{3}\right)^{2}}{2\left(k^{2}+1\right)} d \theta_{3}=\int_{a}^{b} \frac{1}{2} \sin ^{2}\left(\theta_{3}+\phi\right) d \theta_{3}
$$

where $\phi=\tan ^{-1} k \in[-\pi / 2, \pi / 2]$. The cost function $C(k)$ has now been written as a differentiable function of $\phi$ on the interval $[-\pi / 2, \pi / 2]$. It then follows that $C$ has a minimum value on this closed interval and that this minimum occurs either at a point where the first derivative of $C$ with respect to $\phi$ is zero or at an endpoint of the interval. Setting $d C / d \phi$ to zero and applying the second derivative test results in the following optimal solution:

$$
\phi^{*}= \begin{cases}-\frac{1}{2}(a+b)+n \pi, & \text { if } 0<b-a<\pi \\ -\frac{1}{2}(a+b)+\frac{2 n+1}{2} \pi, & \text { if } \pi<b-a<2 \pi\end{cases}
$$

where $n$ is chosen so that $\phi^{*} \in[-\pi / 2, \pi / 2]$. Since (57) is periodic with respect to $\phi$ with period $\pi$, one does not need to check the endpoint $-\pi / 2$ and $\pi / 2$ to know that (58) determines a global minimum. The corresponding $k^{*}$ is found by taking the tangent of $\phi^{*}$ resulting in

$$
k^{*}= \begin{cases}-\tan \left(\frac{a+b}{2}\right), & \text { if } 0<b-a<\pi \\ \cot \left(\frac{a+b}{2}\right), & \text { if } \pi<b-a<2 \pi .\end{cases}
$$

Note that infinite values of $k^{*}$ are allowable and that this in fact does correspond to an inverse that is given by the limit of (48) and (49) as $k$ approaches $\infty$. Thus, for $k=\infty$ the inverse is given by taking $w_{1}=0$ and $w_{2}=-(1 / \sqrt{2}) \cos \theta_{3}$.

To get a more geometrical understanding, note that from (48) and (49) it follows that $w_{2}=-k w_{1}$. Thus, the inverse $G_{r}(\theta, k)$ can be written in the form

$$
G_{r}(\theta, k)=J^{+}+\frac{k \cos \theta_{3}+\sin \theta_{3}}{2\left(k^{2}+1\right)}\left[\begin{array}{c}
\sin \theta_{3} \\
-\cos \theta_{3} \\
1
\end{array}\right]\left[\begin{array}{ll}
1 & -k
\end{array}\right]
$$

where the dependence on the parameter $k$ is made explicit. Note that from (59) it follows that at the center point of a closed interval $[a, b]$ of length less than $\pi$, the optimal inverse is exactly the pseudoinverse. So for such intervals the nearest optimal repeatable inverse matches the pseudoinverse exactly at the interval's center. This optimization can be performed graphically by using the three-dimensional plot shown in Fig. 4. In particular, for $\theta_{3}$ intervals of length less than $\pi$, one would simply choose the $k$ value from Fig. 4 that gives a zero value of $\|\boldsymbol{w}\|_{2}$ at the center of the $\theta_{3}$ interval.

\section{Simulation Results}

In order to illustrate the example in Section IV, this section presents simulation results for the manipulator depicted in Fig. 3 , commanded to follow the 10 square end-effector trajectories shown in Fig. 5. For each square trajectory, the manipulator's initial configuration is set to the origin of the joint space, which corresponds to the point $(x, y)=(1,0)$ in the workspace. The joint space trajectories obtained using pseudoinverse control are shown in Fig. 6. As expected, pseudoinverse control produces a drift in the joint space that results in a set of

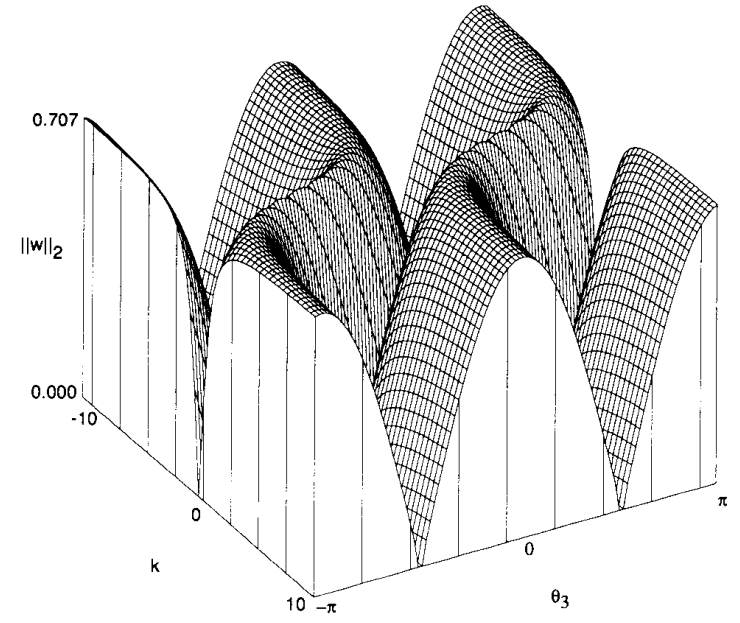

Fig. 4. A 3-D plot of the matrix norm of the difference between the pseudoinverse and the nearest repeatable inverse as a function of $\theta_{3}$ and $k$ for the manipulator depicted in Fig. 3 .

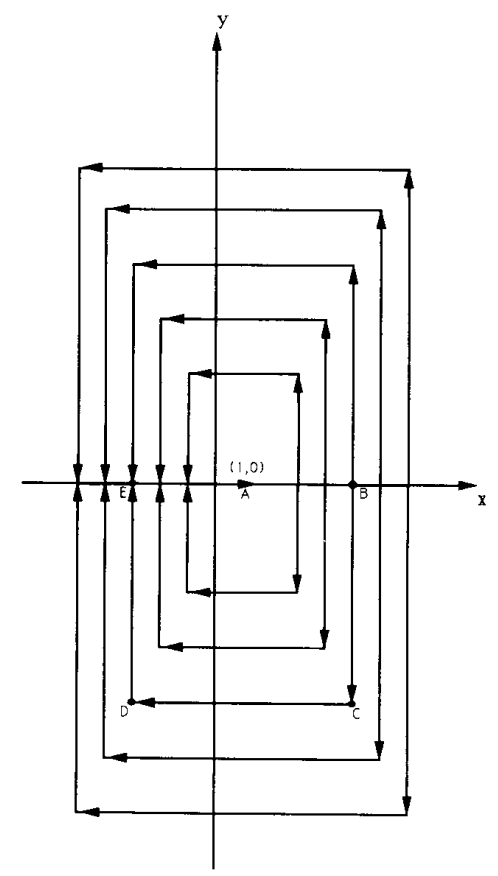

Fig. 5. The end-effector paths used in the simulation of the manipulator depicted in Fig. 3. Each path starts and ends at $(x, y)=(1.0)$.

joint trajectories that spiral along the fiber corresponding to the point $(1,0)$ in the workspace. In Fig. 7 , the same end-effector trajectories and initial conditions are used while applying a nearest optimal repeatable control with $k=0$, which is designed to exactly match the pseudoinverse at the origin of the joint space. The surface corresponding to the value $k=0$ and the initial condition $\left(d_{1}, d_{2}, \theta_{3}\right)=(0,0,0)$ can be clearly seen in this figure. In Fig. 8 , the pseudoinverse trajectory corresponding to the end-effector trajectory denoted 


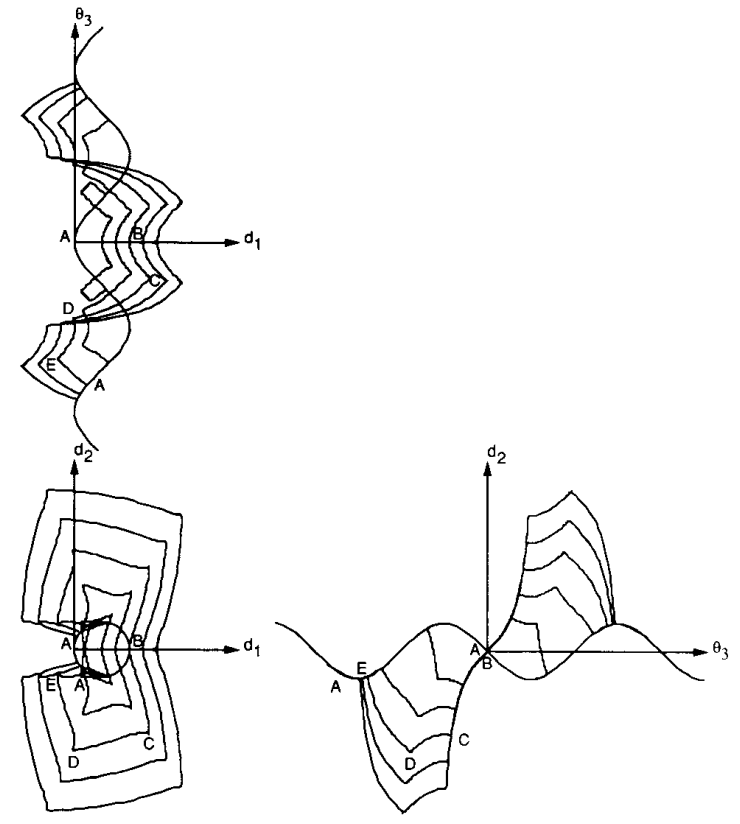

Fig. 6. Three orthogonal views of the ten joint space trajectories obtained when using pseudoinverse control on the manipulator in Fig. 3 to follow the end-effector trajectories shown in Fig. 5. In all cases the initial joint configuration corresponds to the origin. The spiral represents the fiber of the initial end-effector position so that all points on this spiral correspond to the point $(x, y)=(1.0)$ in the workspace. Note the drift in each trajectory.

by $A B C D E$ is shown relative to this integral surface. Note that the pseudoinverse trajectory initially lies on this stable surface, as designed, but starts to diverge as the end-effector leaves point $C$. It is at this point that the global repeatability requirement forces the repeatable inverse to abandon the optimal pseudoinverse solution.

It is clear from Fig. 8 that the constraint of repeatability will restrict the region in which the repeatable inverse can accurately approximate the optimal pseudoinverse solution. However, as shown in the previous section, one does have the ability to select the point at which the approximation is exact by setting the value of $k$ and the manipulator configuration appropriately. This is illustrated in Fig. 9 where two different repeatable controls are compared to the desired pseudoinverse solution. One of the repeatable controls is that used in Figs. 7 and 8 , i.e., with $k=0$ and an initial configuration corresponding to $\theta_{3}=0$, which matches the pseudoinverse at the origin of joint space. The other repeatable control is designed to match the pseudoinverse at the configuration that corresponds to $\theta_{3}=-5 \pi / 4$, denoted by $C$ is the figures, which occurs near the end of the trajectory and requires that $k=-\tan (-5 \pi / 4)=1$. Obviously, the performance of these two inverses is quite different and, due to the harsh constraint of repeatability, they only approximate the desired optimal behavior of the pseudoinverse in their respective design regions. This is more clearly illustrated in Fig. 10, which in effect plots the configuration of the manipulator, since any joint value uniquely identifies the configuration, along the specified end-effector trajectory. It is easy to see from this figure that

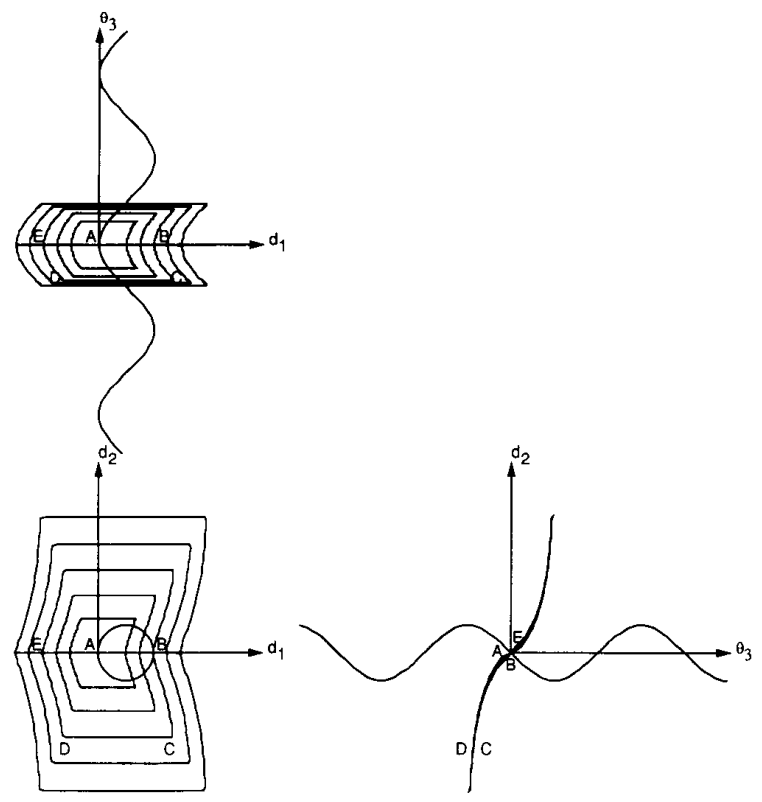

Fig. 7. Three orthogonal views of the ten joint space trajectories obtained when using a repeatable control on the manipulator in Fig. 3 to follow the end-effector trajectories shown in Fig. 5. The repeatable inverse corresponds to $k=0$ and each trajectory has its initial joint configuration at the origin. Once again, the spiral represents the points in the joint space corresponding to the point $(x, y)=(1.0)$ in the workspace. Note that each trajectory returns to the initial starting configuration $\left(d_{1}, d_{2}, \theta_{3}\right)=(0,0,0)$. These trajectories clearly show the surface determined by the initial condition and $k$ value of the repeatable inverse.

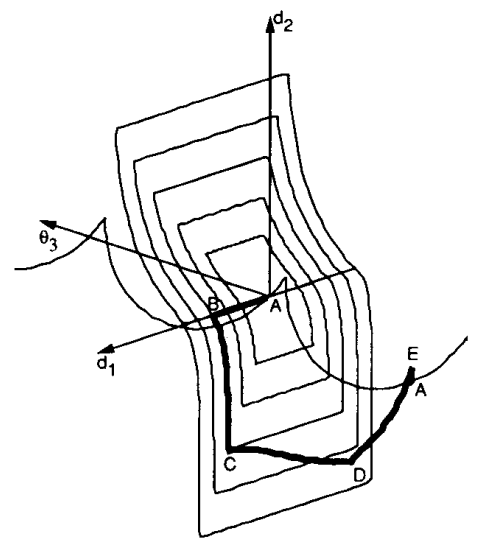

Fig. 8. A 3-D view of one of the joint space trajectories corresponding to pseudoinverse control and the surface representing the repeatable control for the manipulator in Fig. 3 with $k=0$ and initial configuration $\left(d_{1}, d_{2}, \theta_{3}\right)=(0.0 .0)$. Note that the pseudoinverse trajectory initially starts out on the surface, but later the two diverge. In order for a trajectory to be repeatable, it must stay on the surface. At the point where the pseudoinverse trajectory diverges from the surface, the repeatable control can no longer satisfy staying close to the pseudoinverse while maintaining the repeatability requirement.

the $k=0$ repeatable inverse solution exactly matches the performance of the pseudoinverse trajectory up to the point $F$ and that the $k=1$ repeatable inverse exactly matches the pseudoinverse at the design point $G$ and provides a reasonable 


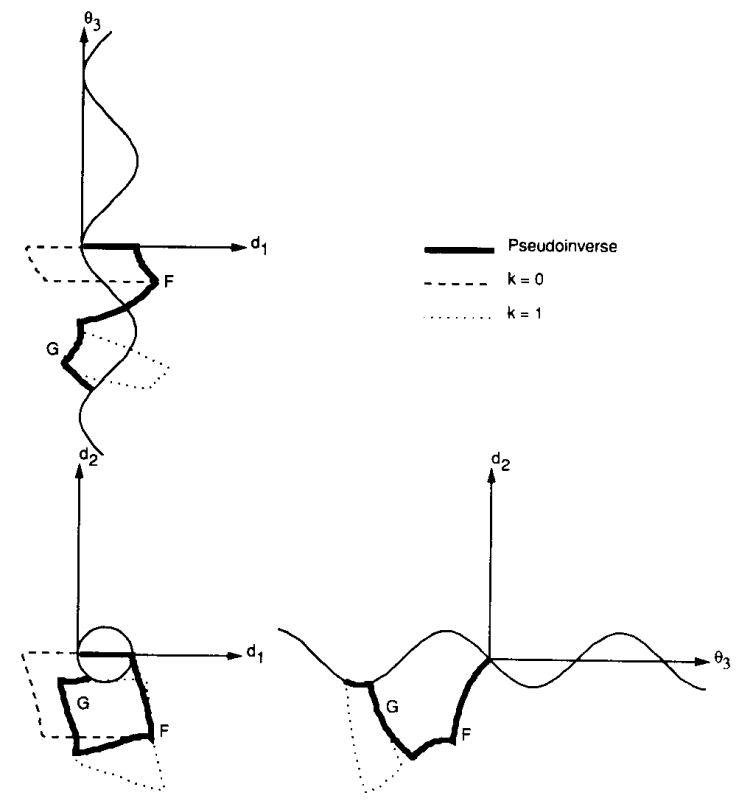

Fig. 9. Three orthogonal views of one joint space trajectory obtained using pseudoinverse control and two trajectories obtained using repeatable controls corresponding to $k=0$ and $k=1$ for the manipulator shown in Fig. 3 . The repeatable trajectories start on the pseudoinverse trajectory at the values $\theta_{3}=0$ and $-5 \pi / 4$, respectively, and illustrate the ability to design a repeatable control that approximates a desired control for a specified region of the workspace.

approximation to the pseudoinverse solution in the region from $D$ to $A$. It is important to note that, despite the fact that the $k=$ 1 inverse configuration and the pseudoinverse configuration match at $H$, this does not imply that the inverses match at this point. This fact is clearly illustrated in Fig. 11 where it can be seen that the joint velocity norm for the $k=1$ solution is considerably higher than the norm of the pseudoinverse solution at point $H$. Fig. 11 also illustrates an important point concerning the "optimal" nature of the pseudoinverse solution, namely, that it is optimal over all possible solutions for a given manipulator configuration. Thus, the apparently anomalous behavior observed near $E$ where the norm of the $k=0$ solution is smaller than the norm of the pseudoinverse solution is not unexpected since the configurations are not identical (see Fig. 10). A pointwise comparison of the joint velocity norm between the $k=0$ solution and the pseudoinverse solution is given in Fig. 12 for identical configurations corresponding to the $k=0$ joint space trajectory in Fig. 9. This figure illustrates the penalty, in terms of increased solution norm, incurred by requiring repeatability. It also illustrates the profound effect that the direction of the specific commanded end-effector velocity has on the difference between the two solution norms. This accounts for the exact match between the pseudoinverse solution and the $k=0$ solution in the region from $A$ to $C$ despite the fact that these two inverses are actually quite different in the region from $B$ to $C$.

\section{CONCLUSIONS}

It has been shown that one cannot rely on the existence

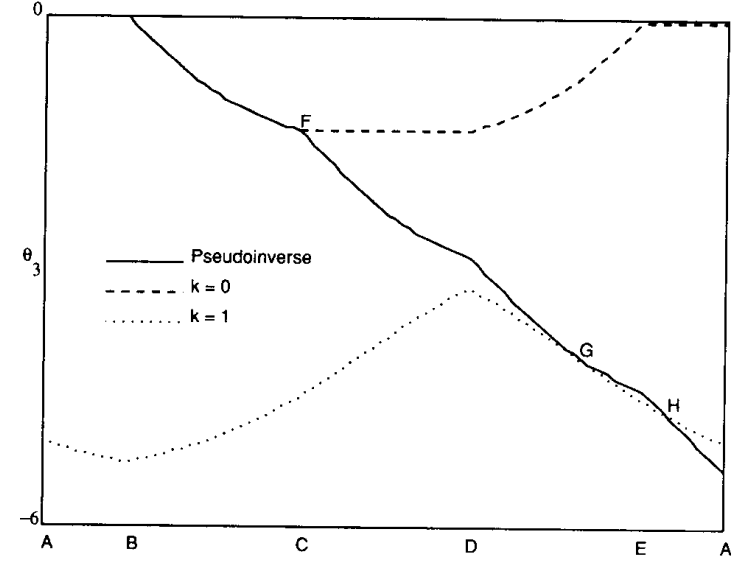

Fig. 10. A plot of $\theta_{3}$ that, together with the end-effector position, uniquely identifies the configuration of the manipulator shown in Fig. 3, as a function of the position of the end-effector in the workspace for the trajectory used in Fig. 9. Point $F$ identifies the configuration at which the repeatable inverse corresponding to $k=0$ diverges from the desired optimal pseudoinverse solution. Point $G$ is the point at which the repeatable inverse corresponding to $k=1$ was designed to exactly match the pseudoinverse solution. Point $H$ identifies a point at which the configurations for both the pseudoinverse solution and the repeatable solution match but whose inverses differ.

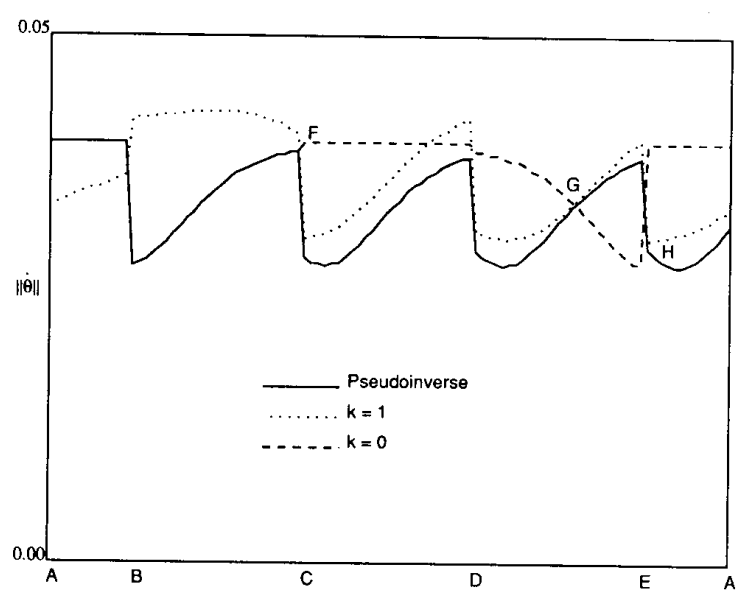

Fig. 11. A plot of the joint velocity norm as a function of the position of the end-effector in the workspace for the trajectory used in Fig. 9. The repeatable inverse corresponding to $k=0$, which was designed to approximate the optimal minimum norm behavior of the pseudoinverse in the initial region of the trajectory, exactly matches its behavior until point $F$. The repeatable inverse corresponding to $k=1$ was designed to approximate the pseudoinverse near point $G$. Note the difference in the solutions at point $H$ despite the fact that the configurations are identical (see Fig. 10). This is due to the fact that the repeatable inverse was not designed to match the pseudoinverse at this point.

of stable surfaces to guarantee repeatable performance for a desired generalized inverse control. The LBC alone, by virtue of the fact that it is only a necessary condition, can only identify candidate surfaces, many of which are not stable surfaces. Likewise, convergence to a particular joint space trajectory for a given end-effector trajectory does not imply the existence of a stable surface. The constraint of repeatability for all end-effector trajectories and all initial conditions, which is characterized by foliations of stable surfaces, significantly 


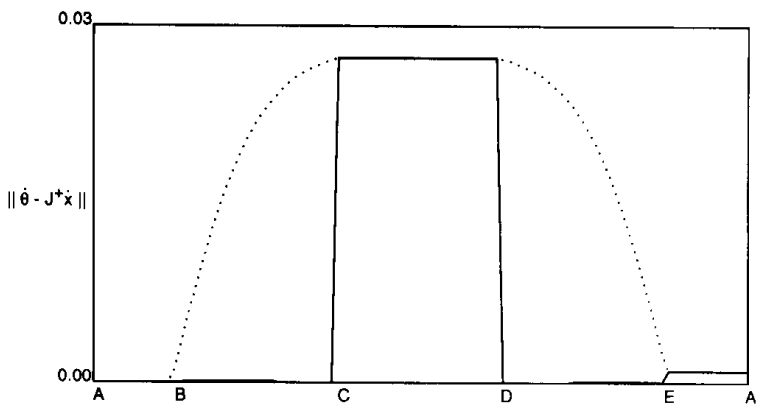

Fig. 12. A plot of the norm of the difference between the joint velocities obtained using the repeatable control with $k=0$ and the pseudoinverse control as a function of the position of the end-effector in the workspace for the trajectory used in Fig. 9 is given by the solid line. The pseudoinverse control is calculated for the manipulator configuration obtained as a result of the selected repeatable control. A plot of $\|\boldsymbol{w}\|_{2}\|\dot{\boldsymbol{x}}\|_{2}$ along the same end-effector trajectory, given by the dashed curve, represents the norm of the worst case difference between the joint velocities of the repeatable $(k=0)$ and pseudoinverse strategies for all trajectories passing through the joint values with an end-effector velocity of $\|\dot{x}\|_{2}=0.04$.

restricts the choice of available generalized inverse controls. However, it has been shown that it is possible to approximate the behavior of any desirable optimal inverse in a specified region by determining the repeatable inverse that is closest to the desired inverse. This results in a control that takes advantage of the available redundancy to locally optimize some desirable performance criterion in the specified region of the workspace while also satisfying the extremely restrictive global constraint of repeatability.

\section{APPENDIX I}

\section{APPLYING THE LBC TO $J^{T}$ INSTEAD OF $J^{+}$}

Shamir and Yomdin [9] established a necessary and sufficient condition for an inverse $G$ to be repeatable. This condition is summarized by the following theorem.

Theorem 1: Let $G$ be the control matrix, which is assumed to be a smooth function in an open subset $\Phi$ of the joint space. Let $U \subset f(\Phi)$ be a simply connected region of the workspace. Then the control is repeatable for any closed paths lying in $U$ if and only if the following condition holds: For any two columns $\boldsymbol{g}_{i}$ and $\boldsymbol{g}_{j}$ of $G$, their Lie bracket $\left[\boldsymbol{g}_{i}, \boldsymbol{g}_{j}\right]$ is a linear combination of the columns of $G$.

Theorem 1, which is called the Lie bracket condition (LBC), provides an analytical tool for testing the repeatability of a given control strategy; however, this technique results in some rather tedious work, which motivates the result given in Proposition 1, which can sometimes simplify the algebraic computations involved. This result basically says that two control strategies that have identical column spaces for each value of $\theta$ in an open subset $\Phi$ simultaneously satisfy or fail the LBC.

Proposition 1: Suppose $L=G T$ on an open subset $\Phi$ of the joint space where $T$ is a smooth nonsingular matrix function. Then $G$ satisfies the LBC on $\Phi$ if and only if $L$ does.

Proof: $(\Rightarrow)$ Let $G=\left[\begin{array}{lll}\boldsymbol{g}_{1} & \cdots & \boldsymbol{g}_{m}\end{array}\right], L=\left[\begin{array}{lll}\boldsymbol{l}_{1} & \cdots & \boldsymbol{l}_{m}\end{array}\right]$, and $T=\left[t_{i j}\right]$. Suppose that $G$ satisfies the LBC on $\Phi$. The matrix $L$ is given by

$$
L=G T=\left[\begin{array}{lll}
\sum_{p=1}^{m} t_{p 1} \boldsymbol{g}_{p} & \cdots & \sum_{p=1}^{m} t_{p m} \boldsymbol{g}_{p}
\end{array}\right]
$$

The Lie bracket of columns $i$ and $j$ of the matrix $L$ is given by

$$
\begin{aligned}
{\left[\boldsymbol{l}_{i}, \boldsymbol{l}_{j}\right] } & =\left[\sum_{p=1}^{m} t_{p i} \boldsymbol{g}_{p}: \sum_{q=1}^{m} t_{q j} \boldsymbol{g}_{q}\right] \\
& =\sum_{p=1}^{m} \sum_{q=1}^{m}\left[t_{p i} \boldsymbol{g}_{p}, t_{q j} \boldsymbol{g}_{q}\right] .
\end{aligned}
$$

From the following identity

$$
[\alpha \boldsymbol{x}, \beta \boldsymbol{y}]=\alpha \beta[\boldsymbol{x}, \boldsymbol{y}]+\alpha(\nabla \beta \cdot \boldsymbol{x}) \boldsymbol{y}-\beta(\nabla \alpha \cdot \boldsymbol{y}) \boldsymbol{x}
$$

where $\alpha$ and $\beta$ are scalar functions and $\boldsymbol{x}$ and $\boldsymbol{y}$ are vector functions, it follows that

$$
\begin{array}{r}
{\left[\boldsymbol{l}_{i}, \boldsymbol{l}_{j}\right]=\sum_{p=1}^{m} \sum_{q=1}^{m}\left(t_{p i} t_{q j}\left[\boldsymbol{g}_{p}, \boldsymbol{g}_{q}\right]+t_{p i}\left(\nabla t_{q j} \cdot \boldsymbol{g}_{p}\right) \boldsymbol{g}_{q}\right.} \\
\left.-t_{q j}\left(\nabla t_{p i} \cdot \boldsymbol{g}_{q}\right) \boldsymbol{g}_{p}\right)
\end{array}
$$

Clearly this is in the column space of $G$ and is thus in the column space of $L$ so that the LBC holds for $L$.

$(\Leftrightarrow)$ Suppose that $L$ satisfies the LBC on $\Phi$. Since $L=G T$ and $T$ is nonsingular, then $G=L T^{-1}$ and the result follows from the only if part above.

Q.E.D.

As a corollary of the above result, one need only apply the $\mathrm{LBC}$ to $J^{T}$ when testing the pseudoinverse for repeatability. A proof is given below.

Corollary 1: Let $\Omega$ be an open simply connected singularityfree subset of the joint space. The LBC holds for $J^{+}$on $\Omega$ if and only if it holds for $J^{T}$.

Proof: Since $J$ is nonsingular on $\Omega, J^{+}$is given by $J^{T}\left(J J^{T}\right)^{-1}$. The result then follows directly from the proposition.

Q.E.D.

A proof similar to the one shown above is given in [5], which appeared while this paper was in review.

\section{APPENDIX II \\ PRoOF OF THE EQUiVALENCE OF THE LBC AND THE CURL CONDITION FOR $\mathbb{R}^{3}$}

For planar three-link manipulators, the LBC can be written in terms of a curl condition. While the LBC utilizes the column space of the inverse $G$, the simpler curl condition, which is given by (6), uses only the null space of $G^{T}$. The equivalence of these two conditions is shown in Proposition 2.

Lemma 1: $G$ satisfies the LBC on an open singularity free subset $\Phi$ if and only if for all nonzero vectors $\boldsymbol{n}_{G} \in \operatorname{ker}\left(G^{T}\right)$

$$
\boldsymbol{n}_{\boldsymbol{G}}{ }^{T}\left[\boldsymbol{g}_{i}, \boldsymbol{g}_{j}\right]=0, \quad 1 \leq i, j \leq m
$$


Proof: $(\Rightarrow)$ Suppose $G$ satisfies the LBC. Then $\left[\boldsymbol{g}_{i}, \boldsymbol{g}_{j}\right]$ is in the column space of $G$. Therefore, there exists a vector function $z$ such that

$$
\left[\boldsymbol{g}_{i}, \boldsymbol{g}_{j}\right]=G \boldsymbol{z}
$$

so that

$$
\boldsymbol{n}_{\boldsymbol{G}}{ }^{T}\left[\boldsymbol{g}_{i}, \boldsymbol{g}_{j}\right]=\boldsymbol{n}_{\boldsymbol{G}}^{T} G \boldsymbol{z}=0
$$

for all $\boldsymbol{n}_{\boldsymbol{G}} \in \operatorname{ker}\left(G^{T}\right)$.

$(\Leftarrow)$ Suppose that (B1) is satisfied for all $\boldsymbol{n}_{\boldsymbol{G}} \in \operatorname{ker}\left(G^{T}\right)$. Then $\left[\boldsymbol{g}_{i}, \boldsymbol{g}_{j}\right]$ is in the column space of $G$ for $1 \leq i, j \leq m$. Therefore, the LBC holds for $G$.

Lemma 2: Let $X: \mathbb{R}^{3} \rightarrow \mathbb{R}^{3}$ and $\alpha: \mathbb{R} \rightarrow \mathbb{R}$. Then

$$
\alpha \boldsymbol{X} \cdot \nabla \times(\alpha \boldsymbol{X})=\alpha^{2} \boldsymbol{X} \cdot \nabla \times \boldsymbol{X} .
$$

Proof: See [10].

Proposition 2: Let $\boldsymbol{n}_{G}$ be any nonzero null vector of $G=\left[\begin{array}{ll}\boldsymbol{g}_{1} & \boldsymbol{g}_{2}\end{array}\right] \in \mathbb{R}^{3 \times 2}$ and let $\Omega$ be an open singularity-free subset of the joint space. Then the following are equivalent:

1) The LBC holds for $G$ on $\Omega$.

2) $\boldsymbol{n}_{G}^{T}\left[\boldsymbol{g}_{1}, \boldsymbol{g}_{2}\right]=0$ on $\Omega$.

3) $\boldsymbol{n}_{G} \cdot \nabla \times \boldsymbol{n}_{G}=0$ on $\Omega$.

Proof: The equivalence of 1 and 2 has already been shown in Lemma 1. It thus suffices to show that 2 and 3 are equivalent. Let $\boldsymbol{g}_{3}=\boldsymbol{g}_{1} \times \boldsymbol{g}_{2}$.

$$
\begin{aligned}
\nabla \times \boldsymbol{g}_{3} & =\nabla \times\left(\boldsymbol{g}_{1} \times \boldsymbol{g}_{2}\right) \\
& =\boldsymbol{g}_{1} \nabla \cdot \boldsymbol{g}_{2}-\boldsymbol{g}_{2} \nabla \cdot \boldsymbol{g}_{1}+\left(\boldsymbol{g}_{2} \cdot \nabla\right) \boldsymbol{g}_{1}-\left(\boldsymbol{g}_{1} \cdot \nabla\right) \boldsymbol{g}_{2} \\
& =\boldsymbol{g}_{1} \nabla \cdot \boldsymbol{g}_{2}-\boldsymbol{g}_{2} \nabla \cdot \boldsymbol{g}_{1}-\left[\boldsymbol{g}_{1}, \boldsymbol{g}_{2}\right]
\end{aligned}
$$

so that

$$
\boldsymbol{g}_{3} \cdot \nabla \times \boldsymbol{g}_{3}=-\boldsymbol{g}_{3}^{T}\left[\boldsymbol{g}_{1}, \boldsymbol{g}_{2}\right]
$$

Thus, $\boldsymbol{g}_{3} \cdot \nabla \times \boldsymbol{g}_{3}=0$ if and only if $-\boldsymbol{g}_{3}^{T}\left[\boldsymbol{g}_{1}, \boldsymbol{g}_{2}\right]=0$. Any null vector $\boldsymbol{n}_{G}$ has the form $\alpha \boldsymbol{g}_{3}$ for some scalar function of $\alpha$. Thus, by Lemma 2 , it follows that $\boldsymbol{n}_{G}^{T}\left[\boldsymbol{g}_{1}, \boldsymbol{g}_{2}\right]=0$ if and only if $\boldsymbol{n}_{G} \cdot \nabla \times \boldsymbol{n}_{G}=0$

Q.E.D.

A proof similar to the one shown above is given in [5], which appeared while this paper was in review.

\section{REFERENCES}

[1] J. Baillieul, "Kinematic programming alternatives for redundant manipulators," in Proc. IEEE Int. Conf. Robotics Automat. (St. Louis, MO, Mar. 25-28, 1985), pp. 722-728.

[2] D. R. Baker and C. W. Wampler II, "On the inverse kinematics of redundant manipulators," Int. J. Robotics Res., vol. 7, no. 2, pp. 3-21, Mar./Apr. 1988.

[3] C. A. Klein and C. H. Huang, "Review of pseudoinverse control for use with kinematically redundant manipulators," IEEE Trans. Syst. Man Cybern., vol. SMC-13, no. 3, pp. 245-250, Mar./Apr. 1983.

[4] C. A. Klein and K. H. Kee, "The nature of drift in pseudoinverse control of kinematically redundant manipulators," IEEE Trans. Robotics Automat., vol. 5, no. 2, pp. 231-234, Apr. 1989.

[5] S. Luo and S. Ahmad, "Measure of joint path drift for kinematically redundant robots," in Proc. IEEE Int. Conf. Robotic Automat. (Sacramento, CA, May 1991), pp. 1163-1168.

[6] A. A. Maciejewski and R. G. Roberts, "Utilizing kinematic redundancy in robotic systems: Practical implementations and fundamental limitations," in Proc. Amer. Contr. Conf. (San Diego, CA, May 23-25, 1990), pp. 209-214.

[7] H. Seraji, "Configuration control of redundant manipulators: Theory and implementation," IEEE Trans. Robotics Automat, vol. 5, no. 4, pp. 472-490, Aug. 1989.

[8] T. Shamir, "Remarks on some dynamical problems of controlling redundant manipulators," IEEE Trans. Automat. Contr., vol. 35, no. 3, pp. 341-344, Mar. 1990.

[9] T. Shamir and Y. Yomdin, "Repeatability of redundant manipulators: Mathematical solution of the problem," IEEE Trans. Automat. Cont. vol. 33, no. 11 , pp. 1004-1009, Nov. 1988.

[10] I. N. Sneddon, Elements of Partial Differential Equations. New York: McGraw-Hill, 1957.

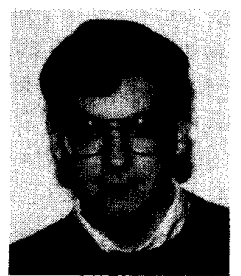

Rodney G. Roberts (S'91) received the B.S. degrees in electrical engineering and mathematics from the Rose-Hulman Institute of Technology, Terre Haute, IN, in 1987 and the M.S.E.E, and Ph.D. degrees from Purdue University, West Lafayette, IN in 1988 and 1992, respectively.

His research interest is in the motion control of kinematically redundant manipulators.

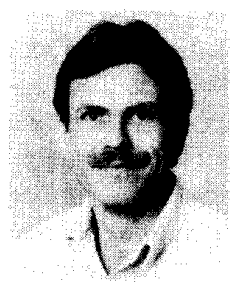

Anthony A. Maciejewski (S'82-M'87) received the B.S.E.E., M.S., and Ph.D. degrees in electrical engineering from The Ohio State University, Columbus, in 1982, 1984, and 1987, respectively.

Since 1988, he has been an Assistant Professor with the School of Electrical Engineering at Purdue University, West Lafayette, IN. His primary research interests center on the simulation and control of kinematically redundant systems. 